(e) Want of the diversity in the pressure-symptoms met with in the malignant growths.

(f) An obscure sense of fluctuation.

The recognition of a mediastinal fatty tumor would be attended with difficulty, enhanced by the fact that sarcoma of this region may attain considerable size and lead to a fatal issue, without grave impairment of the general nutrition of the body. A woman, aged 60 years, short of stature and enormously fat, died in my ward at the Jefferson Hospital, of sarcoma of the anterior mediastinum, the symptoms of which had lasted almost eighteen months. Kronlein ${ }^{1}$ described a congenital lipoma of the anterior mediastinum, in a child aged one year, which found its way through an intercostal space to the surface of the chest, and then rapidly increased in size.

When we come to consider the differentiation of the malignant new-growths which constitute the more common tumors of this region, we find that a probable diagnosis of lymphoma is warranted

(a) When the tumor is primary.

(b) When there are evidences of widespread disease of the lymphatic glands, with or without enlargement of the spleen.

(c) The younger the subject, the greater the probability that the tumor is lymphomatous.

Carcinoma is rendered probable by

(a) The previous or coincident occurrence of cancerous disease in the mammæ or elsewhere :

(b) The presence of hard, nodular, immovable masses in the neck:

(c) Relatively slow development of the tumor :

(d) A tendency to rapid emaciation in the absence of evidences of direct pressure upon the cesophagus.

(e) The occurrence of cachexia.

(f) The intensity and knife-like character of the pain :

(g) A relatively advanced period of life.

(h) A hereditary predisposition to carcinomatous disease.

If the evidence in favor of the other forms be wanting, the diagnosis of sarcoma may be reached by exclusion. This view would be rendered more probable by

(a) Rapidity of growth ;

(b) The history of the amputation of a limb, resection of a joint, or extirpation of any organ for sarcomatous disease.

All these forms of malignant growth are alike liable to be followed by secondary invasion of the lung.

I have purposely omitted, in the foregoing paper, the consideration of many of the multifarious phenomena of pressure and invasion met with in mediastinal tumors. The necessity to be brief has obliged me to simplify the subject by serious omissions. I find a further excuse, also, in the fact that each case must be worked out not so much by fixed rules of procedure, as by careful and painstaking analysis of its symptoms.

\footnotetext{
2 Langenbeck, Klinic, p. 157 .
}

\section{LIMITATIONS OF PATHOGNOMONIC SIGNS AND SYMPTOMS.}

\author{
BY EDWARD G. JANEWAY, M.D., NEW YORK.
}

Read in the Section on Practice of Medicine and Materia Medica of American Medical Association, May, 1884.

It has been the writer's intention to bring into this short paper, the results of observations extending over some time concerning certain limitations which must be placed upon signs which are considered, when present in certain states, more or less pathognomonic of a definite morbid condition. The word pathognomonic is not used in a strict sense, but somewhat as the word diagnostic. The writer has so frequently witnessed erroneous opinions as to the nature of disease, arising from the reception of some one sign as absolutely determining, that he has been impelled to write this article to impress more forcibly the necessity of studying all the phenomena present in cases of disease. Those diagnoses which are rightly denominated snap, may at times be brilliant, but he who indulges in them frequently, no matter how well informed, will be sure to make mistakes. Diseases do not follow an absolute rule in their manifestations, and he who would have them always square with the written description will obtain but a partial insight into their nature. As the time allotted is brief, I shall only allude to some of the facts of the nature indicated by the title, and not attempt to cover the whole field of medicine. Moreover, I shall restrict it to this consideration as regards signs.

The nervous system allows of fewer claims of pathognomonic signs or symptoms than do most of the other organs. Yet we find that there is a tendency to accept certain states as proving the existence of a given disease. It was not so long since that many were willing to accept the presence of optic neuritis or choped disc conjoined with headache as characteristic of a cerebral tumor; and the change in intelligent medical opinion on this subject shows how the ground shifts with an increasing attention paid to a subject. At present we would accept the optic neuritis as indication of increased intracranial pressure only, not necessarily that this pressure was due to a tumor; moreover we should place the limitation to this consideration by excluding the possibility of Bright's disease, or of this as capable of explaining all the phenomena of a given case. The writer has on a number of occasions found that those who were thoroughly conversant with the examination of the eye, would alter the opinion expressed as to the cause of an optic neuritis as between disease of the kidney, and intra-cranial pressure with the course of the case and the development of symptoms.

Such a symptom or sign can only be accepted with considerable limitation as pathognomonic.

There seems to be a wide discrepancy in this matter, also, as regards the examination of the fundus of the eye, in cases of so-called congestion of the brain. Some have relied upon a certain state of the retinal vessels as proving that the brain was also congested. Yet here, surely, the margin must be very large, for 
the writer has met instances in which patients, who, having the same symptoms during their whole course of medical pilgrimage in New York, and at times abroad, also, have been told that they had congestion by some, anæmia by others. Nor would this be alluded to, in this connection, were it not that some have put forth such extravagant statements upon the certainty of distinguishing in this way these opposed states of the cerebral circulation. Volitional tremor is relied upon, also, as discriminative of multiple sclerosis, yet here we have to consider the possibility of metallic poison, especially mercury, or the effects of alcohol, before accepting the tremor as due to this lesion.

Perhaps the most difficult matter which comes to a physician for solution is coma, and if in this state we could meet with some sign of absolute distinction, we might be delivered from much of the uncertainty of diagnosis. There are those who hold that in this state the temperature of the body will furnish a clue as between uræmia and hæmorrhage; but, unfortunately, some claim an elevated temperature, others a normal, as proper to the former condition. The truth is that we find the temperature too variable to become a guide, though a lowering at the outset, with subsequent elevation, points very certainly, if not with absolute positiveness, to hæmorrhage as the cause of the unconscious state. But we meet here a very decided limitation, in that we so often fail to obtain a knowledge of the initial lowering of temperature.

Others have proposed and would rely upon the presence of albumen and casts, one or both, as distinctive between uræmia and hæmorrhage. Alas! only too frequently disease of the kidney co-exists with cerebral hæmorrhage, so that the presence of albumen and casts are not infrequent accompaniments of the latter of these states, and do not help us in deciding as to the cause of the coma. So, too, if we attempt to find some sign which will distinguish between the different causes productive of hemiplegia, it will soon become apparent that there is no infallible guide. The writer has found those signs which are regarded as pathognomonic, as, for instance, between embolism and hæmorrhage, or obliterating endarteritis, liable at times to mislead, unless their application is limited with certain qualifications. For illustration, heart murmurs may exist, and yet, instead of cerebral embolism, hæmorrhage may be the cause of a hemiplegic attack. The reverse is, also, not infrequently true; a heart murmur is absent, and yet cerebrai embolism takes place, owing to the obstructing plug having been detached from a thrombus out of the way of the direct blood current, as in the auricle, etc. So, too, an obliterating endarteritis may cause a sudden attack; in such case the already narrowed lumen is probably closed by a rapidly formed coagulum, and that suddenness of onset which is often considered pathognomonic of embolism, or hæmorrhage, be simulated by obstruction. Neither does the method of recovery upon which others would depend always lead us safely through the maze of possibilities naturally inherent in these cases.

Again, rigidity of the neck through tonic spasm of the cervical muscles, is a valuable sign in cases where a fever or internal inflammation is liable to be confounded with meningitis. It is, however, in no sense pathognomonic, or, rather, admits of considerable limitation.

Beyond those cases where a slight degree of it is present in pneumonia, etc. (of childhood particularly), the writer has found it very markedly present in one case of ventricular hæmorrhage, and in one case of sub-ependymal softening of the lateral ventricle, involving, to a considerable degree, the sensory tracts in their passage toward the occipital lobes. The case was one recently under observation, having severe headache, neuro-retinitis, cerebral irritability, with increasing apathy, to which this symptom became added.

The writer, as others, has been obliged to receive tendon reflexes, or rather their plus and minus changes, with considerable limitations, as indicative of disease. He has found, as others, the normal possibilities too considerable.

Electrical reactions, though much relied on, do not absolutely discriminate the nature of a lesion nor its site ; for instance if there is degeneration, reaction present, the lesion may either be situated in the nerves, the anterior nerve roots, in the anterior cornua, or be of uncertain site, as in the case of leadpoisoning. A knowledge of the history and causation is essential to a correct appreciation of a given case.

Illustrations could be given were there time.

In considering pulmonary complaints we find but few signs considered pathognomonic of themselves, but some which in connection with others are regarded as determining.

Thus vocal fremitus is often relied upon as distinguishing between pleural effusion and pneumonic consolidation, where a doubt may have existed, owing to the peculiar combination of signs present. That it has a certain value the writer is prepared to admit, but that it can in no sense be considered as positive, the following brief allusions will illustrate. The writer has met two cases of complete consolidation of the lung with obstruction of the main bronchus by an aneurism in which there was absence of vocal fremitus, etc. Again it is not a very rare event to have vocal fremitus preserved in cases of pleural effusion, nay,even to have it intensified. The writer has frequently noted mistakes made by supposing vocal fremitus preserved, because existing at the upper level of pleural effusions, when absent below. There is perhaps no greater difficulty than is presented by a case of this character in which the bronchial breathing is present to a notable degree. Even the exploratory puncture by the hypodermic syringe-needle has its limitations in these conditions, because it might be possible to have it fail of withdrawing fluid owing to obstruction, leakage, etc., or if withdrawing it, to make sure that it is not because of the existence of a combination of the two diseases. It might be added that the writer has examined some of these cases after death, and became assured that both serous effusion and empyema can, independent of a consolidation, cause increase of vocal fremitus. The limitations then in a 
doubtful case become so great that the sign loses a large measure ot its force. The limitations of cracked pot percussion note as distinctive of a cavity are so familiar to you all, that it would be a waste of time to repeat them. There may be some, however, who are not familiar with the want of reliance to be placed upon bronchial breathing as discriminating between pneumonia and pleurisy with effusion. It is a very common thing to have bronchial breathing present in cases of pleural effusion, and it follows a certain method and character. It is usually present at the upper limit and has a somewhat sniffling tone, and gives, as compared with the bronchial of consolidation, the idea of coming from a distance. It may, and then usually with these characters, be present over the whole effusion. At times, however, this bronchial breathing is so plain and concentrated that even the best examiners are misled by it into supposing consolidation to exist.

The writer has noticed this mistake more frequently in empyema and in the pleural effusion of inflammatory rheumatism. 'To make a short digression, the writer will explain one of the causes of this occurring under these circumstances. The patient is hurriedly set up, the physician percusses rapidly, noting dullness or flatness over the lower part of the chest, and then, running his ear over this portion, is struck by finding bronchial breathing, not noting that, as a rule, it is present only at the upper part of the fiat or dull region. The vocal resonance is also often misleading, being broncophonic. Hence in teaching it is wiser to state that bronchial breathing is usually due to consolidation resulting from infiltration, exudation, etc., but at times also to compression of the lung. Limitations too are necessary in considering the importance of the signs considered pathognomonic of a cavity. Probably you are all aware that at times the physical signs of a cavity may exist, and yet the lung be consolidated, or compressed by fluid. The writer has been particularly impressed with this in the examination of certain cases of empyema, serous pleurisy and hydrothorax. Under these circumstances he has heard amphoric breathing, whispering, pectoriloquy, and amphoric percussion note over a space in the lower part of the inter-scapular region, and close to the lower angle of the scapula as a rule, which are extremely deceptive or liable to be so, were the observer not fore-warned by previous experiences. Some of these cases have been followed to the autopsy, others into convalescence, in both series the result showed that these characteristics were simulative of a cavity, not evidences of its existence. No very good explanation could be offered for this deviation from the general rule so far as the writer has been able to study it in the cases which have come under observation. The writer would only draw attention to one point in speaking of the limitation of vocal resonance, because these follow the corresponding breathing states to which allusion has been made, and that is to mention the existence of a normal pectoriloquy of which he has assured himself in a number of cases. His attention was first drawn to it by finding that when describing vocal resonance as heard over normal lung in the usual terms, and then presenting some one as an example, that the description, and the illustration would not match, but instead, that articulate speech was distinctly audible over the chest.

The pathognomonic importance of râles as indicative of fluids in bronchial tubes or of changes in their calibre, or in the case of the crepitant, of separation of the sticky sides of the air cells and small bronchioles is but little called in question. Yet there are those who believe that pleural conditions cause certain of these phenomena. The writer has met a few cases where a crepitant râle was present in the initiation of a pleurisy. Hence, beyond the usual limitation of this râle, he would add this as of rare occurrence in his experience. Under such circumstances he supposes that the separation of the sticky pleural surfaces so takes place as to produce crepitation instead of grazing or rubbing. There are few cases which would support the view that the subcrepitant râle is of pleural origin; pleural exudation may be met with in some cases where subcrepitation existed during life, but the writer has seen numerous cases in which subcrepitant râles were present during life, and no exudation was present on the pleura post mortem.

Another sign regarded as pathognomonic is the succussion sounds. There can be no question of its origin from the shaking of air and fluid in a hollow space. The writer has, however, obtained it in several other conditions than hydro-pneumothorax, notably so in cases when from catarrhal or other trouble fermentation occurred in the stomach. He has, however, met with but one instance in which such succussion had led to a mistake in diagnusis. In that instance pneumonia existed, and the physician in attendance was led to shake the patient by hearing a metallic bubbling, and observing the splashing sounds came to an erroneous conclusion. The stomach succussion requires the use of a little more force and is not in all instances readily audible over the chest though heard at a distance. The limitation which it would be safer to place in teaching this sound would be that it might arise from other spaces containing air and fluid, as the stomach. It might be added, that the writer has obtained it in a fibrocystic tumor of the uterus whose walls were gangrenous, in an ovarian sac having a communication with the exterior, and in the intestines largely distended; and that though he has attempted to produce it the case of large cavities in the lung he has hitherto not succeeded.

In the study of diseases of the heart, several important limitations are to be placed upon murmurs as pathognomonic. Not infrequently the writer has met persons with weak heart from myocarditis, degeneration, etc., who had been informed, presumably by competent authority, that they had no disease, because of the absence of murmurs, and of evidence of marked enlargement of the heart. And on the other hand, it is supposed that because a murmur is heard over the heart, or in such a position as is natural to valvular disease to occasion it, that said murmur is due to valvular disease. You are all aware of anæmia, and of respiration, as at times the cause of mis- 
takes. The writer would draw attention more particularly to the possibility of aneurism, or of arterial degeneration with dilatation causing a murmur in the aorta above the valves, or behind the heart, and transmitted through it so as to simulate aortic or mitral valvular disease. Notwithstanding one is aware of the likelihood of encountering cases of this nature, or that one under consideration may be of this nature, it will be found at times, examining as carefully as possible, extremely difficult to reach a certain decision between such conditions. At least, it has fallen under the writer's observation that such mistakes were made by practiced auscultators, and he has himself been extremely puzzled by some cases of this nature, and come to a right conclusion only after several examinations; and in two which he met some years since, decided upon the wrong condition. One instance in particular impressed him, because of the small amount of trouble. This consisted of a thrombus about $1 / 2$ inch long, situated on the aorta, about an inch above the valves, taking its origin from an ulcerated atheromatous patch. The ante-mortem diagnosis by a clever physician was aortic valvular disease, obstructive and regurgitant; thus showing that the pre-diastolic aortic murmur caused by the recoil of blood on the valves, or rather, the arterial systolic, may be mistaken for aortic regurgitation. If a digression be allowed for a moment, it may be in order to point out the necessity of keeping in mind, in such cases, that a murmur just before, and, as with the second sound, above the valves, whilst there is no murmur at such time or during ventricular diastole, should raise a suspicion, at least, that the murmur is produced in the aorta, and not at the valves. Such a consideration has been of great service to the writer in establishing the proper site of murmurs.

Another sign which the writer has heard laid down as diagnostic as between an aneurism or a tumor pressing on an artery or in the neighborhood of an artery, is the presence of a double arterial murmur, this being regarded by some as positive evidence of an aneurism. The writer has paid careful attention to this matter, and has found it fail so often that he regards the proposition as utterly untenable. $\mathrm{He}$ has had particular occasion to study it in the epigastric region. In four cases of carcinoma of the stomach or lympho-sarcoma in this region, he has found a double murmur present over this area, and in some of these cases, with no pressure exerted by the examiner. The new growth, as the autopsy showed, pressed on the aorta, and at the stenosed portion not only did a murmur occur during cardiac, but also during arterial systole. It should be added, that marked epigastric pulsation was present in all. In a recent case, the writer found such a double murmur due to a partial stenosis of the aorta by connective tissue going over it. The patient had radiating pains from the spine into the anterior crural and lumbar nerves, evincing pressure on these, considerable epigastric pulsation, to which was added this double murmur, particularly audible when the heart was acting strongly. Another limitation is also necessary in the consideration of double arterial murmur, which has likewise been the cause of difficulty to a number of physicians. This consists in the presence of venous puss in the chest and neighborhood of the aorta. In this latter place it is more deceptive than when found in the neck, though even in that situation, especially in men, the writer has found it mistaken for an aneurismal murmur.

A sign which any physician on first impression would be liable to misinterpret is the severe dyspnoa which at times accompanies Bright's disease. Should one encounter a patient with severe subjective and objective dyspncea, having at the same time a considerable degree of cyanosis, he would be liaile, nay almost certain, to say that such patient had some diffculty with the lungs or air passages primarily or secondary to cardiac trouble. Yet in such condition, independent of the effect of externally received poison, we meet an important limitation, one which is not sufficiently familiar to many physicians. This constitutes the dyspnœa of Bright's disease, or, as some say, the nervous dyspnœea of Bright's disease, asthma of Bright's disease. The writer has met a number of such cases. There is no demonstrable action of the heart; no obstruction in the bronchi; the respiratory murmur is perfect, without the least râle, and yet the patient feels in himself and seems to others in danger of dying of suffocation. In fact, when severe dyspnœa exists, for which the condition of the heart and lungs offer no explanation, this Bright's dyspncea should be suspected, and the urine examined for evidences of the disease. The writer would be willing to admit that some cases can be plausibly explained upon the nervous theory, but this explanation will not satisfy the conditions when cyanosis exists. How the nervous system could induce this is a mystery to the writer, considering that the patient may be inspiring and expiring forcibly, and the heart is throwing the blood through the lungs and body (at least there is no proof to the contrary).

The idea which he formed when examining a marked case of this kind, was that by some chemical or other change the red blood globules did not absorb oxygen. All cases in which odema, hydrothorax, etc., exist, are excluded from this category.

The writer will close the paper by drawing attention to two signs upon which it is necessary to place certain limitations. The first is the presence of albumen in the urine. That this may occur from other causes than Bright's disease is probably familiar to all, and that it can be formed in the urine of apparently healthy indiyiduals has been emphasized of late by several observers. The writer knows of no better illustration than one he met a year ago. The patient, a male, broker, had had severe headaches, and on one occasion, for a time, glycosuria. He had had his urine examined in consequence repeatedly, but albumen had not been detected. Coming to the writer's office one morning in consequence of the headache, he was, as is the custorn, asked for a sample of urine. This was found loaded with albumen. The morning and evening urine were requested, but these showed no evidence. A series of experiments were made by the patient, some of which are of sufficient interest to detail. The morning urine would be free, but a cool 
sponge bath, reading a book or mental exercise, would instantly cause a marked albumenuria. At no time during six weeks' observation could the writer detect casts, or prove that the kidneys were otherwise affected than to allow from different causes albumen to transude. A re-examination after a lapse of six months showed the same condition.

So also casts, especially the hyaline, may be present in the urine for a long time without thereby proving a serious lesion of the kidneys. It is possible that casts may, under certain conditions, form in normal kidneys.

The writer has recently had occasion to examine the urine of a gentleman who has a considerable number of casts, hyaline or slightly granular, coming only after severe exercise. The urine is normal in quantity, specific gravity, and has not contained albumen. The writer has examined the urine passed at other times of the same day on several occasions with negative results, except the presence of mucus and occasionally mucous casts. Only too frequently we are apt to alarm a patient by the detection of what may be considered a sign of imperfect action, yet not necessarily of confirmed or incurable disease, and to pronounce them afflicted with a malady whose very name is a terror.

These illustrations and remarks will suffice for the end had in view, though the same line of consideration could be continued indefinitely.

\section{THE USE OF ANTISEPTICS IN PUERPERAL CASES.}

BY MADISON REF.CE, M.D., OF ABINGDON, ILL.

[Read before the Military Tract Medical Association.]

During one of the hottest days of the month of July, I 881, I was called to see Mrs. W., near London Mills, in consultation with Dr. Wilson, of that place. I found her in a small bed-room, only large enough for the bed upon which she was lying, with but one window in the room. She had been confined a week previously. Her pulse was $\mathrm{I} 60$, her temperature $106^{\circ}$, the tongue dry, abdomen distended to the size of a woman at full term, the secretion of milk suppressed. She was delirious, and an odor filled the room like that of a body far gone in putrefaction.

The husband was informed that there was no probability whatever for the recovery of his wife, every symptom indicating a speedy dissolution. However, I suggested a thorough washing out of the uterus with antiseptics. A half gallon of warm water with two drachms permanganate of potash was prepared. After being placed in a proper position across the bed, a gum catheter of the largest size, with several fenestræ in the lower end, was passed up into the uterus as far as it would go. A common Davidson syringe was attached to it by means of a piece of rubber tubing. The uterus was thoroughly washed out, the injection being continued until the water returning was perfectly free from all odor and clear in color. The discharge from the uterus was of such a pecu- liarly strong odor, that it was impossible to free the hands from it. It was like that one acquires in the dissection of bodies.

Such a change as took place in this woman's case within an hour, I never before saw in any case. Immediately after this cleansing process the delirium passed off, the temperature fell, the pulse came down -in short, the effect was simply magical. She was given twenty grain doses of the sulphite of soda. The husband was taught how to administer the injections, which were to be given every three hours-one of a solution of permanganate of potash, the other of carbolized water, alternately. The patient made a speedy recovery from the severest attack of puerperal septicæmia I have ever seen.

Twelve years before this case,. in September, I 869, I was called to see a woman who had had an abortion produced by mechanical means. She had a rapid pulse, high fever, hot skin, unquenchable thirst, irregular chills, an icteric countenance, and a sanious discharge from the uterus that smelled horribly.

A half dozen intra-uterine injections of carbolized water thoroughly administered, rescued her from impending death.

Ever since that time I have used this method in all such cases with similar results with a few exceptions. They are to be regarded as similar to surgical fever, the result of decomposing débris in the uterus, which is absorbed from the uterine surface, and which Sir James Simpson has aptly compared to the flap of an amputated thigh, with its raw and bleeding surfaces, and patulous vessels ready to absorb decomposing fluids with which they may lie in contact.

The following long-continued case illustrates, in another way, the value of these intra-uterine injections. The last day of January, saw Mrs. M. A., aged 24 , living near Hermon, a strong and vigorous woman. She had been confined 24 hours previously. The child was dead when delivered, and was so much decomposed that portions of the skin slipped from the body when handled. She had had a chill and profuse flooding a few hours before I saw her. The pulse was I 40, the temperature $105^{\circ}$, skin hot, tongue dry, and abdomen tympanitic and distended to the size it was before her confinement.

The uterus was immediately washed out with carbolized water, giving great relief to the patient. The injections were kept up every three hours for a week, with the effect of reducing the temperature after every injection. Quinine was also freely administered. Thinking, perhaps, that the septic matter was sufficiently removed from the uterus, the injections were discontinued. Within 24 hours a severe chill, followed by a high temperature, announced the absorption of more septic material. The intra-uterine injections were again resorted to, with the same results, diminishing of temperature, and lowering of the pulse. At the end of a week, as there seemed to be so much improvement, they were once more discontinued. Again, in a short time, there was a chill and return of former symptoms. Again we returned to the use of the intra-uterine injections, and kept them up until every vestige of fever had disappeared from the case for several days. 